\title{
Design of Multiregional Supervisory Fuzzy PID Control of pH Reactors
}

\author{
Shebel AlSabbah, ${ }^{1}$ Mujahed AlDhaifallah, ${ }^{1,2}$ and Mohammad Al-Jarrah ${ }^{1}$ \\ ${ }^{1}$ College of Engineering at Wadi Aldawaser, Prince Sattam bin Abdulaziz University, P.O. Box 54, Wadi Aldawaser 11991, Saudi Arabia \\ ${ }^{2}$ College of Computer Science and Engineering, King Fahd University of Petroleum and Minerals, Dhahran 31261, Saudi Arabia \\ Correspondence should be addressed to Shebel AlSabbah; shebel_asad@hotmail.com
}

Received 3 May 2015; Revised 24 June 2015; Accepted 26 July 2015

Academic Editor: Ai-Guo Wu

Copyright (C) 2015 Shebel AlSabbah et al. This is an open access article distributed under the Creative Commons Attribution License, which permits unrestricted use, distribution, and reproduction in any medium, provided the original work is properly cited.

\begin{abstract}
This work concerns designing multiregional supervisory fuzzy PID (Proportional-Integral-Derivative) control for $\mathrm{pH}$ reactors. The proposed work focuses, mainly, on two themes. The first one is to propose a multiregional supervisory fuzzy-based cascade control structure. It would enable modifying dynamics and enhance system's stability. The fuzzy system (master loop) has been chosen as a tuner for PID controller (slave loop). It takes into consideration parameters uncertainties and reference tracking. The second theme concerns designing a hybrid neural network-based $\mathrm{pH}$ estimator. The proposed estimator would overcome the industrial drawbacks, that is, cost and size, found with conventional methods for $\mathrm{pH}$ measurement. The final end-user-interface (EUI) front panel and the results that evaluate the performance of the supervisory fuzzy PID-based control system and hybrid NN-based estimator have been presented using the compatibility found between LabView and MatLab. They lead to conclude that the proposed algorithms are appropriate to systems nonlinearities encountered with $\mathrm{pH}$ reactors.
\end{abstract}

\section{Introduction}

In order to overcome the nonlinearity issue found with most real plants, a wide variety of linear control systems have been developed [1]. PID controllers and conventional algorithms are the most popular control methods used in industry [1-3]. Nevertheless, they are proper for a specific operation range with a linearized plant model. Whenever perturbations lead the process to work out of its operating point, manual adjustment of PID controller parameters is required. Different approaches have been developed to deal with such issue, like predictive model based and neural networks [1]. In [4-7], the utilization of fuzzy logic as online PID tuner has been proposed. Fuzzy control systems are able to supervise the controller performance in the steady state and transition state. Despite this, such algorithms may have poor dynamic performance at certain operating points. To enlarge the working points that are covered by the controlled plant, a multiregional supervisor control is being considered in this work, as will be explained on the coming sections [7].
Our primary motivation is to build end-user-interface (EUI) using the compatibility found between LabView and MatLab. The designed EUI would provide a flexible testbed for modeling and implementation of advanced control strategies without the expense or danger of working with real-time processes.

In this paper, there are two main contributions:

(i) The first one is the development of a multiregional supervisory fuzzy PID (MSF-PID) system to improve the accuracy and modify the dynamics of the PID-controlled $\mathrm{pH}$ process. The proposed algorithm has been designed with cascade structure.

The proposed fuzzy-based control works in subdivided regions. These regions are derived from the preknowledge experience about input-output pattern of the corresponding process. It is concerned with adjusting the gains of PID control with respect to parameter uncertainties and environmental conditions.

(ii) The second contribution concerns proposing an alternative and nonconventional estimation method of process 


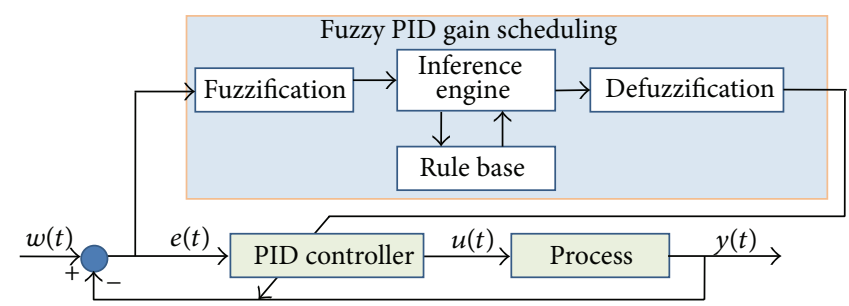

FIGURE 1: Structure of supervisory fuzzy PID.

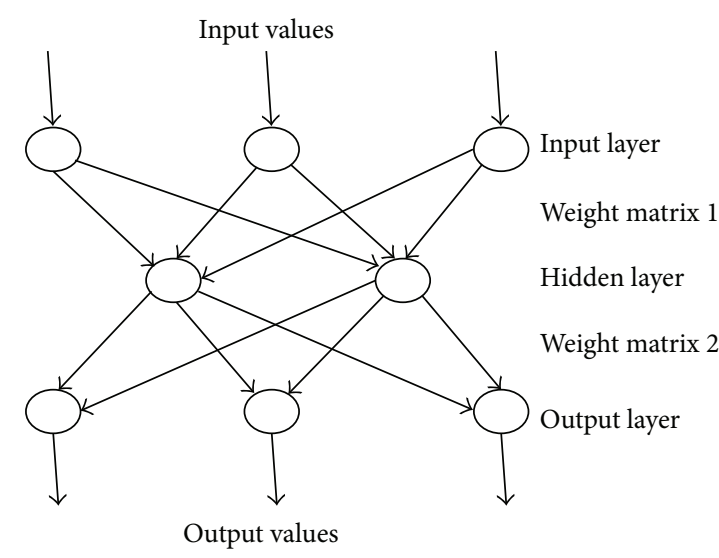

(a)

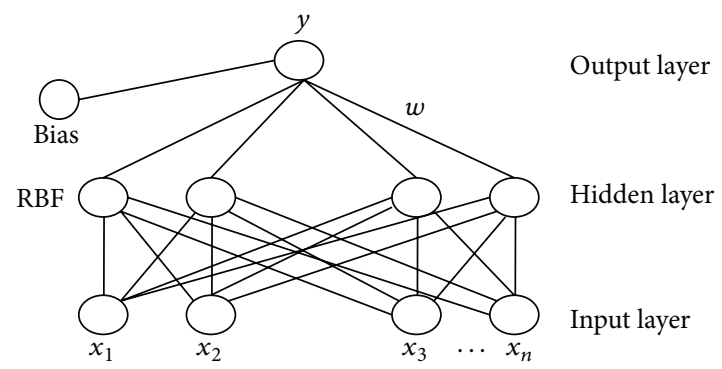

(b)

FIGURE 2: Structure of the proposed neural networks ((a) MLP and (b) RBF).

output. Using the compatibility features found between multilayer perceptrons (MLP) and radial basis function (RBF) neural networks, a hybrid NN-based (HNN) estimator is developed. This intelligence-based estimator would replace the real hardware so that it ensures a compact size and a reduced cost when comparing with the conventional methods of measurement. It will also compensate the errors we might encounter with conventional methods of measurement $[8,9]$.

\section{Preliminaries}

2.1. Supervisory Fuzzy Controller. Supervisory fuzzy controller is a hierarchical one with the supervisor at the highest level, as shown in Figure 1. The fuzzy supervisor can use any available data from the control system to characterize the system's current behavior so that it knows how to change the controller and ultimately achieve the desired specifications. In addition, the supervisor can be used to integrate other information into the control decision-making process.

Figure 1 shows the supervisory fuzzy PID where $u(t)$ is the control action and $y(t)$ is the process output.

The adjustment of PID parameters is carried out by some candidate rules as follows:

(i) If steady-state error is large then increase the proportional gain.

(ii) If the response is oscillatory then increase the derivative gain. (iii) If the response is sluggish then increase the proportional gain.

(iv) If the steady-state error is too big then adjust the integral gain.

(v) If the overshoot is too big then decrease the proportional gain.

In some applications, controller gains are quantified according to different types of responses a priori identified from experiments on the real process [7].

2.2. Hybrid Neural Network. The hybrid structure of neural networks (HNNs) consists of MLP and RBF. Figure 2 shows the structures of RBF and MLP. The main difference between MLP and RBF is that, unlike the MLP, there is only a hidden layer in RBF network which contains nonlinear nodes called RBF units that measure the distance between an input data vector and the center of their RBF [8].

The MLP and RBF networks are trained using a supervised training rule which attempts to minimize the error between the network and the target output patterns. If target outputs are not required for training, the learning rule is unsupervised and the network extracts its own features from the training set.

For choosing the optimal and adequate structure, certain number of neuronal architectures would be studied. Different initialization of synaptic parameters has been done for each architecture to ensure that the training of the NN converges towards the least error criterion. For each structure, the mean 


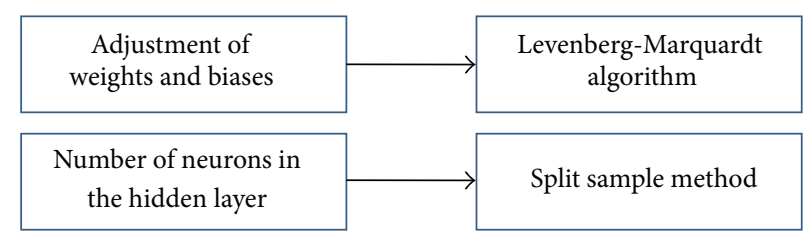

FIGURE 3: Training and parameters adjustment.

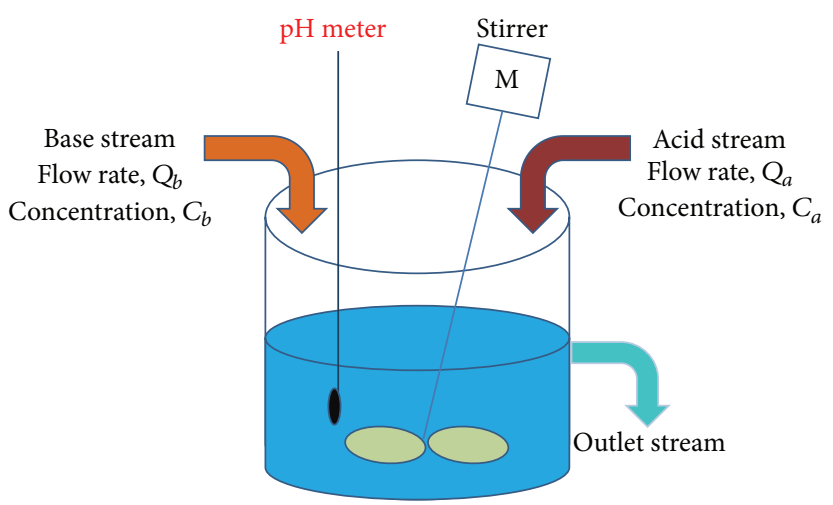

Figure 4: $\mathrm{pH}$ reactor.

square error of the training and validation databases could be calculated. Then, the optimal structure is the structure which has the least square error in the validation base. The training had been done using Levenberg-Marquardt algorithm [8]. The training is carried out on a field of study called "field of training." We create three databases belonging to the field of training: training, validation, and testing database, as in Figure 3.

\section{3. pH Reactor: Modeling, Controlling, and Estimation}

Figure 4 demonstrates the $\mathrm{pH}$ reactor considered in this work. It is one of the most demanded processes in different industrial sectors such as food and diary, medicine, and biomedical industry $[10,11]$.
3.1. Model of $p H$ Reactor. The logarithmic relation between the base streams as manipulated variable and the $\mathrm{pH}$ value as a master process variable has been studied, as will be seen in Section 3.2. This relation is subdivided into three operating zones (two linear ranges and one nonlinear range). It aims, mainly, to enlarge operating range of controlling $\mathrm{pH}$ and modify its close-loop response.

In our case, the considered titration process has a strong acid (HCL) with a constant flow value $[2 \mathrm{~mL} / \mathrm{sec}]$, where its concentration is equal to $[0.95 \mathrm{~mol} / \mathrm{L}]$. The manipulated variable will be the flow of strong base $(\mathrm{NaOH})$, with a concentration $[1.9 \mathrm{~mol} / \mathrm{L}]$. At this level, $\mathrm{pH}$ would be calculated using the following equation $[1,7]$ :

$$
\mathrm{pH}=\log _{10}\left[\frac{-\left(k Q_{a} C_{a} /\left(Q_{a}+Q_{b}\right)-Q_{b} C_{b} /\left(Q_{a}+Q_{b}\right)\right)+\sqrt{\left(k Q_{a} C_{a} /\left(Q_{a}+Q_{b}\right)-Q_{b} C_{b} /\left(Q_{a}+Q_{b}\right)\right)^{2}-4\left(10^{-14}\right)(-1)}}{2\left(10^{-14}\right)}\right]
$$

where $C_{a}, C_{b}$ are the concentration values of acid and base, respectively, in the outlet stream, $Q_{a}, Q_{b}$ are the volumetric flow rates of acid and base, respectively, $V$ is the volume, and $k$ is a constant that depends on the strength of acid. In our case, $k=1$ is considered for strong acid-strong base system $[7,10]$.

3.2. MSF-PID Control of $p H$ Reactor. In this subsection, a modified cascade structure of MSF-PID control system is proposed to meet the industrial control demands due to its nonlinearities which interfere with gain adjustment of the process.

Figure 5 shows the block diagram of the $\mathrm{pH}$-based fuzzy supervisory control system. As it can be seen from the block diagram, the fuzzy system takes three inputs, auxiliary variable $\left(\mathrm{pH}^{*}\right)$, error in $\mathrm{pH}$ value $(e)$, and change in error $(d e)$, and generates three outputs: proportional gain $K_{P}$, integral gain $K_{I}$, and derivative gain $K_{D}$. $U$ is the control action; $Q_{b}$ is the manipulated variable and represents the flow of base. 


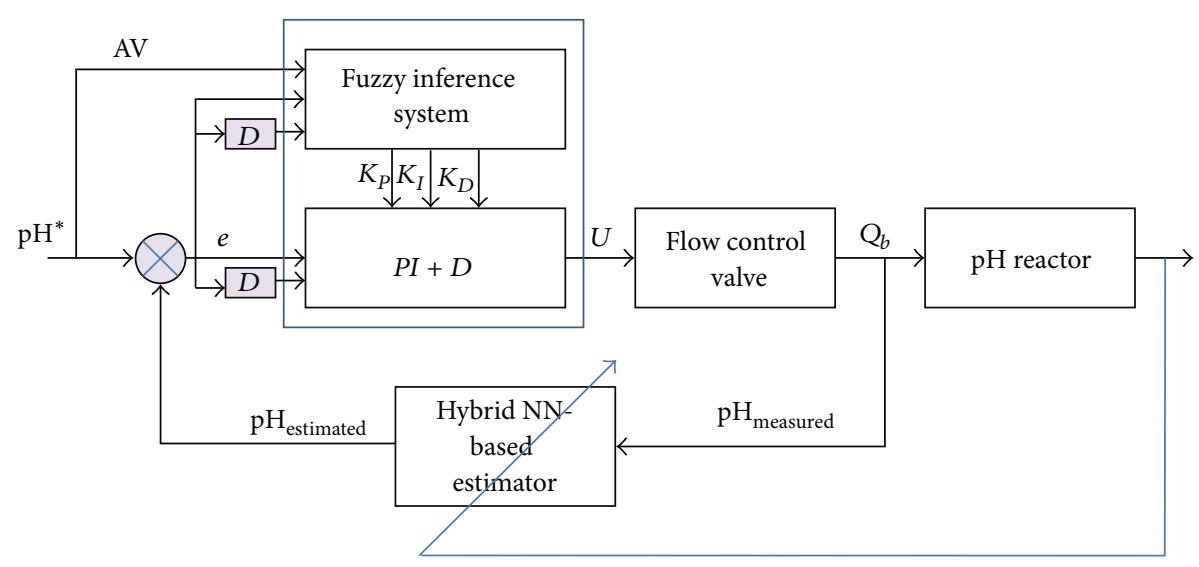

FIGURE 5: Supervisory fuzzy PID-based control over $\mathrm{pH}$ reactor.

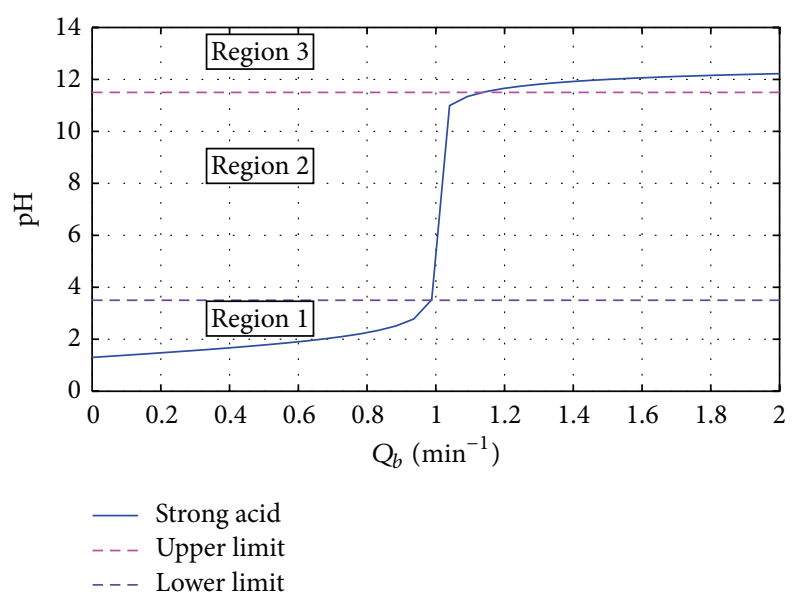

FIgURE 6: The titration curve subdivided into three regions.

The proposed control system is working on three subdivided titration regions. These three regions have been chosen with respect to the value of base flow rate $Q_{b}$ that manipulates the $\mathrm{pH}$ value inside the reactor, Figure 6.

Since the variables and their state are linguistic values, they can only be interpreted qualitatively and inexactly. Therefore, a technique is needed to describe these vague values. Fuzzy set is one of the perfect tools to process the linguistic information.

Regarding structure of proposed fuzzy, there are three inputs to fuzzy inference: auxiliary input $\mathrm{pH}^{*}$ (region 1: low; region 2: medium; and region 3: high), error $e$ and derivative of error $d e$, and three outputs which are $K_{P}, K_{I}$, and $K_{D}$. The PID controller has two inputs (control error and derivative of error).

Figures 7(a) and 7(b) show the membership functions of all the inputs and outputs. They are composed of different shapes (triangular and Gaussian) and sizes to accomplish all linear and nonlinear features found with such process dynamics (Figure 6) and also to ensure smooth operation around set point (modified dynamics) and a minimum steady-state error (enhanced stability).
The widths of the fuzzy sets used for controllers are not the same and they have been determined by trial and error experience. The width of the fuzzy sets for $K_{P}$ has been chosen [ 0.20 .7$]$, for $K_{I}$ is [ 0.0010 .01$]$ and for $K_{D}$ is [ 0.10 .15$]$. And for inputs, the range of set point as an auxiliary input $\mathrm{pH}^{*}$ is $\left[\begin{array}{ll}0 & 14\end{array}\right]$, the range for the error has been chosen $\left[\begin{array}{ll}-1 & 1\end{array}\right]$, and for error rate is $[-1010]$. The auxiliary input is used to precisely localize at which region $\mathrm{pH}$ varies.

The designed fuzzy tuner would upgrade the values of $K_{P}, K_{I}$, and $K_{D}$ upon to the fuzzy inputs ( $e$, de as measured inputs and the set point as AV). The structure of the classical controller is supposed to be $P I+D$. Two tables would be supplied (Tables 1(a) and 1(b)), as an example, to show the fuzzy rules with respect to two measured inputs ( $e$ and $d e$ ) and a fixed set point ( $\mathrm{AV}=$ medium $)$, since the medium state of the third input means that the process runs in region 2 (nonlinear region) as seen in Figure 6. The AV is used to specify where $\mathrm{pH}$ is located (linear or nonlinear regions).

Table 1(a) shows the fuzzy rules which are used to determine the fuzzy outputs $\left(K_{P}\right.$ and $\left.K_{I}\right)$ with respect to measured inputs; these rules would ensure the enhanced stability with minimum residual error at the final state.

Table 1(b) shows the fuzzy rules used to determine $K_{D}$. In Table 1(b), the rules have been designed in a way where the best dynamic features (smooth rising time and least overand undershooting) could be assured with the presence of derivative control action.

Table 1 presents only the rules with respect to measured inputs ( $e$ and $d e$ ). It is also showing the influence of measured inputs on PID control gains of the proposed structure $(P I+$ $D)$. But the set point (AV) is also necessary to specify the region of operation (linear or nonlinear, Figure 6).

3.3. HNN-Based pH Estimator. One of the main objectives of this paper is the design and application of a numerical $\mathrm{pH}$ estimator integrated into titration process as an industrial replacement of real hardware electrodes to measure $\mathrm{pH}$. The proposed estimator is designed with LabView and MatLab. First, the MLP and RBF are used separately to design $\mathrm{pH}$ estimator. Then, a hybrid NN structure is developed to accomplish the best features found in both MLP and RBF. The 


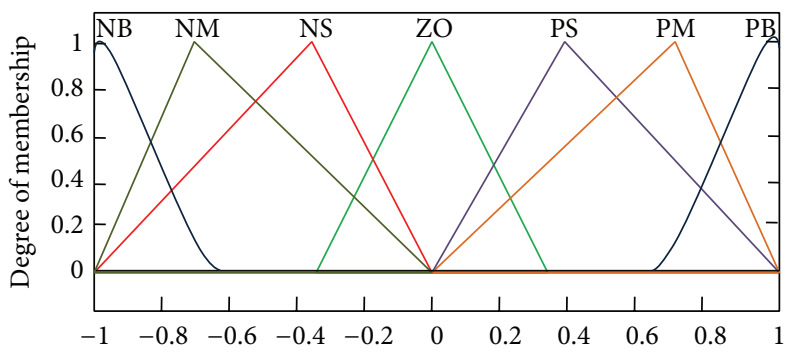

(a)

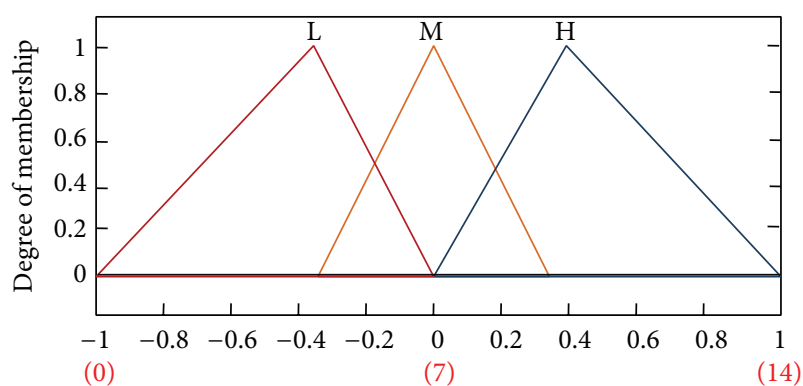

(b)

FIGURE 7: Membership functions for (a) $e$, de, $K_{P}, K_{I}$, and $K_{D}$; (b) AV with normalised discourse.

TABLE 1: Fuzzy rules at medium auxiliary variable (AV is $\mathrm{M})$ as a case study.

(a)

\begin{tabular}{ccccccccc}
\hline & \multicolumn{8}{c}{ Error } \\
\hline & & NL & NM & NS & ZO & PS & PM & PL \\
& NL & ZO & NS & NM & NL & NM & NS & ZO \\
& NM & PS & ZO & NS & NM & NS & ZO & PS \\
& NS & PM & PS & ZO & NS & ZO & PS & PM \\
Error rate & ZO & PL & PM & PS & ZO & PS & PM & PL \\
& PS & PM & PS & ZO & NS & ZO & PS & PM \\
& PM & PS & ZO & NS & NM & NS & ZO & PS \\
& PL & ZO & NS & NM & NL & NM & NS & ZO \\
\hline
\end{tabular}

(b)

\begin{tabular}{ccccccccc}
\hline & \multicolumn{7}{c}{ Error } \\
\hline \multirow{5}{*}{ Error rate } & NL & NM & NS & ZO & PS & PM & PL \\
& NL & ZO & PS & PM & PL & PM & PS & ZO \\
& NM & NS & ZO & PS & PM & PS & ZO & NS \\
& ZO & NM & NS & ZO & PS & ZO & NS & NM \\
& PS & NM & NS & ZO & PS & ZO & NS & NM \\
& PM & NS & ZO & PS & PM & PS & ZO & NS \\
& PL & ZO & PS & PM & PL & PM & PS & ZO \\
\hline
\end{tabular}

split-sample method is implemented to select the optimal NN structure.

A conventional $\mathrm{pH}$ measurement loop is made up of three components: the $\mathrm{pH}$ sensor, which includes a measuring electrode, a reference electrode, and a temperature sensor; a preamplifier; and an analyzer or transmitter. The measuring electrode, which is sensitive to the hydrogen ion, develops a potential (voltage) directly related to the hydrogen ion concentration of the solution $[12,13]$.

Many troubles of conventional $\mathrm{pH}$ measurement could be faced in practice, for example, electrical interference, relay hunting, in-line calibration, current transmission 4$20 \mathrm{~mA}, \mathrm{pH}$ measurement in liquids with hydrofluoric acid, prevention of chemical wastage, and manual temperature compensation [13].
To overcome the drawbacks which might be found with conventional method for measuring $\mathrm{pH}$, technical and commercial ones, an HNN-based $\mathrm{pH}$ estimator has been proposed. The HNN estimator aims to achieve high accuracy and hardness and treat the nonlinearity of titration curve.

The main structural difference between MLP neural network and RBF one is that, in MLP, the main function is a tansig in first layer but in RBF the main function is Gaussian in latest layer; this makes RBF-NN better than MLP-NN in nonlinear stages of titration process.

The testing database is of different values than the precedent ones (training and validation database). The error between the real and observed $\mathrm{pH}$ values is defined for each parameter by the relative error $(\mathrm{RE})(\mathrm{pH})$ as illustrated in Figure 8.

Figure 8 shows the computed relative error (RE) at both nets. It seems that the RE produced by MLP-NN is $1.82 \%$ which is larger than that of RBFNN (0.133\%). That is why the MLPNN is recommended to be used with linear regions in the titration process and the RBFNN with nonlinear region, as will be seen later.

Referring to the multiple regions established on titration curve (Figure 6), it can be noted that the RBF network would deal tightly with region 2 (fast variation) when $Q_{b}$ varies from $0.8 \mathrm{lt} / \mathrm{min}$ to $1.2 \mathrm{lt} / \mathrm{min}$, and the MLP-NN would ensure the rabidity when dealing with the linear regions (1 and 3 ).

Figure 9 shows the performance of the proposed hybrid net, where it can be seen that the allowance of the sumsquared error (SSE) has been reached $\left(10^{-14}\right)$ with 11 epochs. So both the accuracy and the speed enquiries have been achieved.

With this estimator, the industrial costs could be reduced when replacing the real hardware with numerical hybrid structure connected to the base stream (flow transmitter), and the size could be also reduced. So, the work could match the commercial benefits, when realized.

\section{Simulation with LabView/MatLab}

4.1. Design Steps. The process has one manipulated variable which is the flow of base $Q_{b}$. Inside the process, a scalar circuit has been designed that has two values ( 0 or 1 ) while $Q_{b}$ is limited between 0 and 2 .

The design process is divided into three steps. 

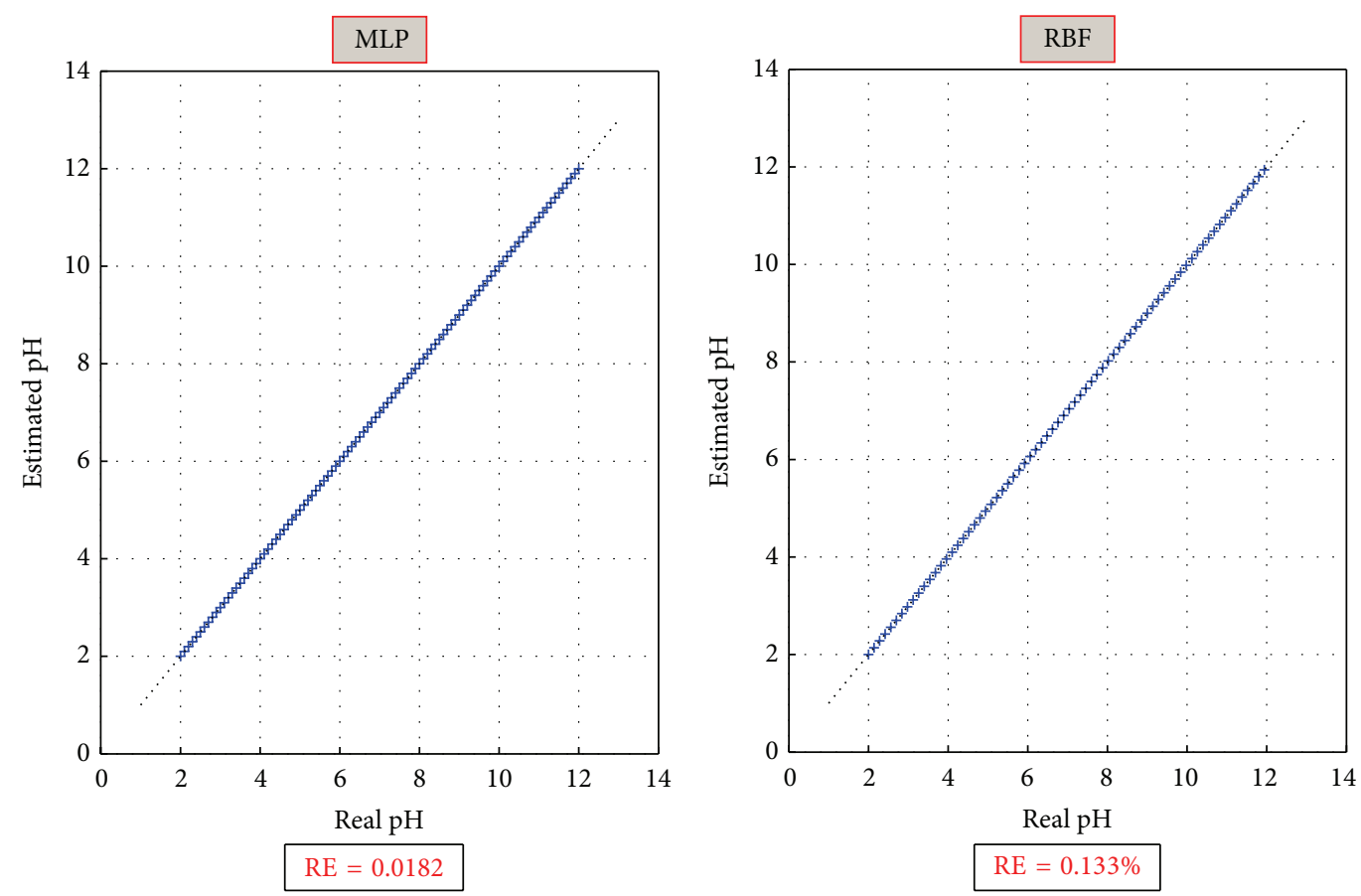

FIGURE 8: The computed relative error (RE) for both RBFNN and MLPNN.

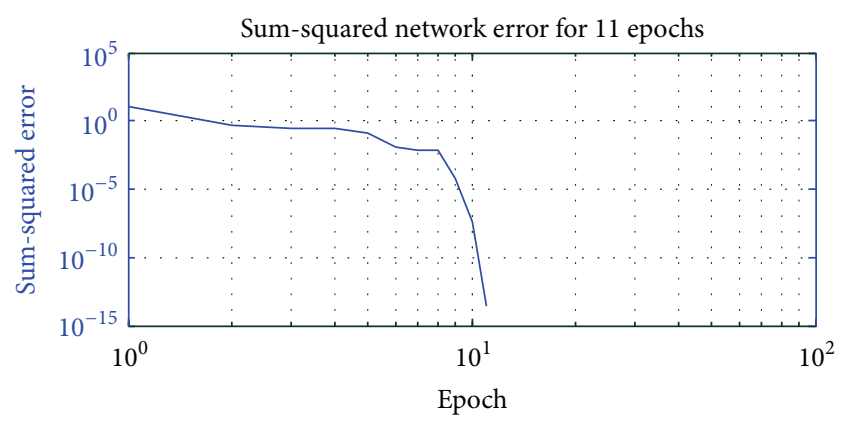

FIGURE 9: The SSE plot for the performance of the hybrid NN-based estimator.

First step is determining the valve position from 0 to 1 ; then, the position of valve returned to the input of the process and multiplied by the flow again to give a new flow value and a new valve position. This step will be repeated periodically during the life time of system.

Second step is determining the ions of hydrogen $\mathrm{H}^{+}$by proposing an inverse model of the process. The result from this process is returned partially as a feedback to insure the continuity of the system.

Third step is designing the mixer block diagram; as mentioned previously, the inputs of mixer are (diameter, length, flow, and initial height), while it has two outputs which are flow out and level.

4.2. EUI. Using the compatibility found between LabView and MatLab (M-files) as programming languages, the HNNbased $\mathrm{pH}$ estimator has been designed, Figure 10.
The final EUI has been created (Figure 11) to facilitate users' studying and analyzing of $\mathrm{pH}$ reactor and titration process at different control strategies.

\section{Results}

The comparative results of PID controller and supervisory fuzzy PID controller within the three regions of the titration curve and towards the set point tracking are presented in Figures 12, 13, and 14. Figure 12 shows the response of PIDcontrolled $\mathrm{pH}$ reactor. Because of the decrement in the static gain of the process, the dynamic response becomes slower for operating point superior to 9.8 (70\%).

In Figure 13, the overshoots and undershoots were smaller than 5\%. It appears more satisfactory than that shown in Figure 12.

The auxiliary input, $\mathrm{pH}^{*}$, has been used as an additional fuzzy input to generate new results that modifies the overall performance of the control system. Figure 14 shows the performance of multiregional supervisory fuzzy PID controller with set point tracking $\left(\mathrm{pH}^{*}: 4,8,12\right)$ at three operating regions in titration process for weak acid and strong acid.

Figures 12,13, and 14 clearly show a superior performance of the multiregional fuzzy PID-based cascade controller over the $\mathrm{pH}$ reactor with minimal resulting errors.

\section{Evaluation of Results}

The performance of the proposed multiregional supervisory fuzzy-based controller has been evaluated and compared with conventional PID-based algorithm. The evaluation was performed using the integral of absolute error (IAE) and 


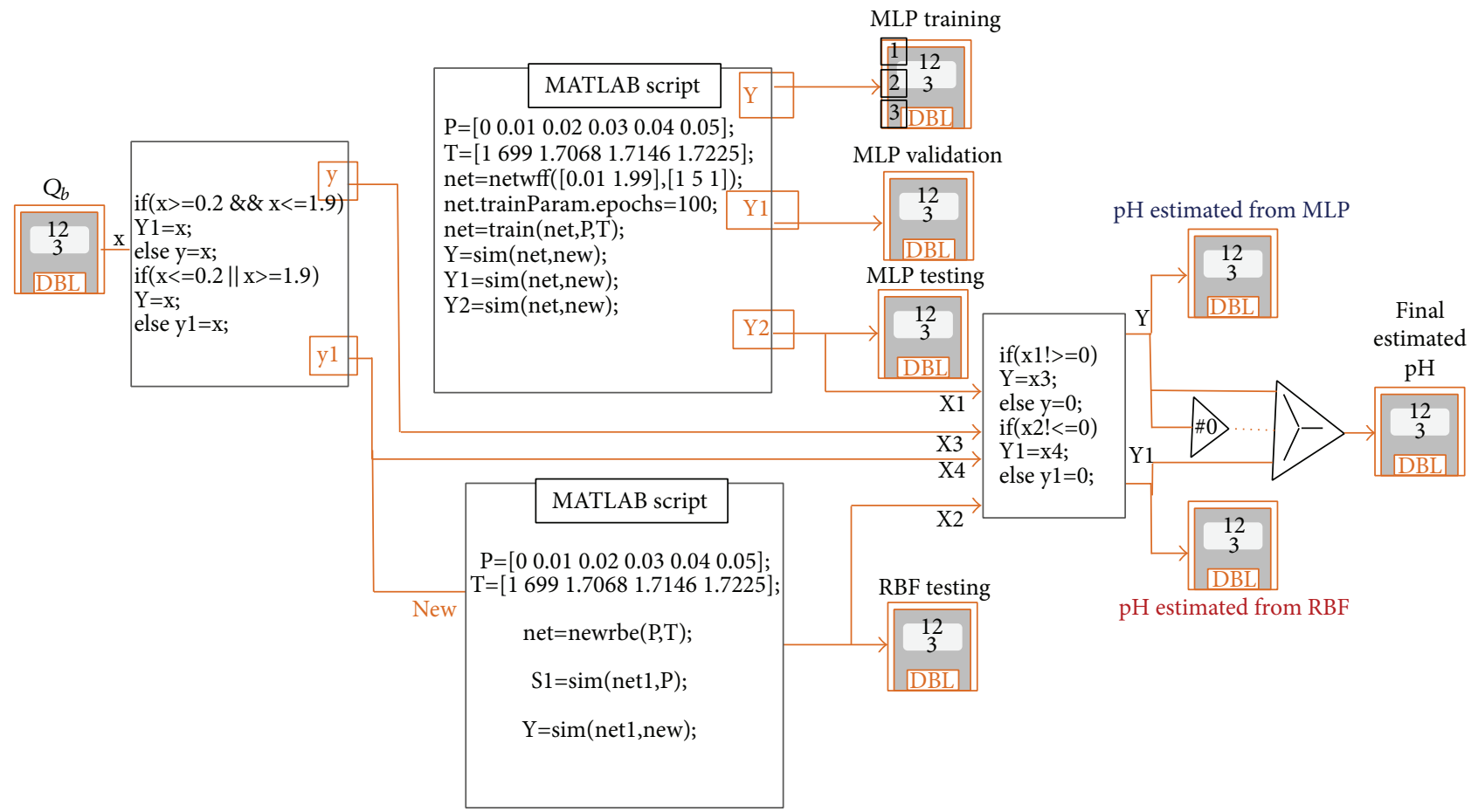

Figure 10: The MatLab script files of HNN in LabView.

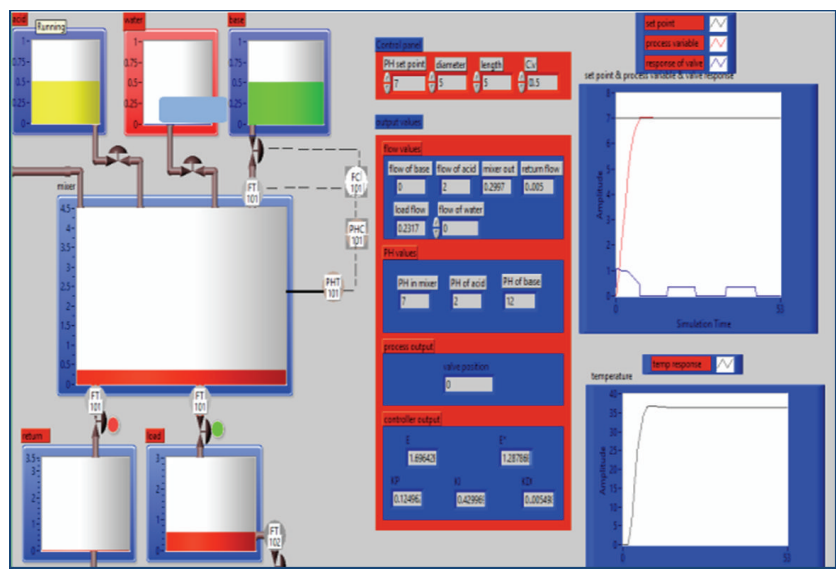

FIgURE 11: The final end-user-interface (EUI).

integral of the absolute value of the error-weighted time (ITAE), as given in Tables 2 and 3 .

From Tables 2 and 3 , it could be clearly noticed that the multiregional supervisory fuzzy PID is better in performance than PID at all reference points.

\section{Conclusions and Future Work}

\subsection{Conclusions}

(i) The nonlinear behavior exhibited by the $\mathrm{pH}$ process was tested using multiregional supervisory fuzzy PID-based cascade control. It is proved to be

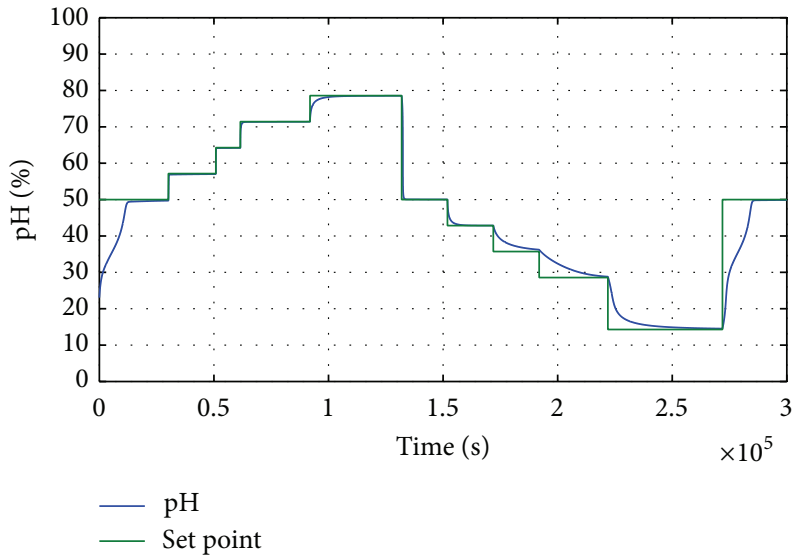

FIGURE 12: PID-controlled response.

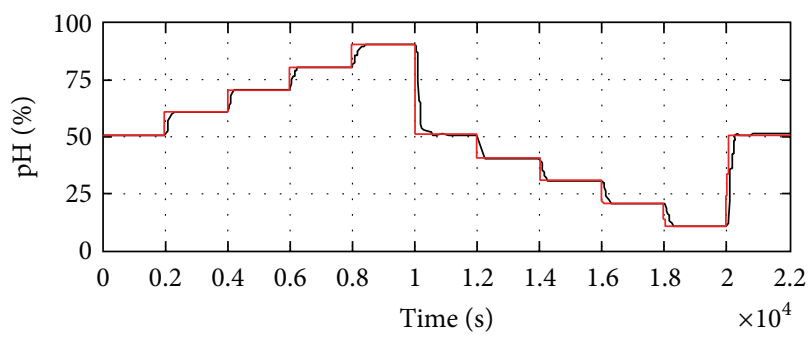

FIGURE 13: Supervisory fuzzy PID response process.

smoother and more robust than classical PID ones that suffer from problems of parameter tuning. 


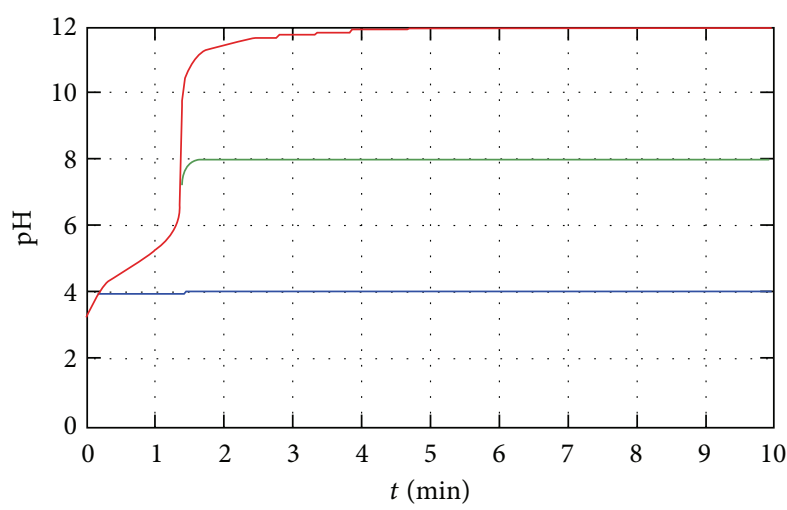

(a)

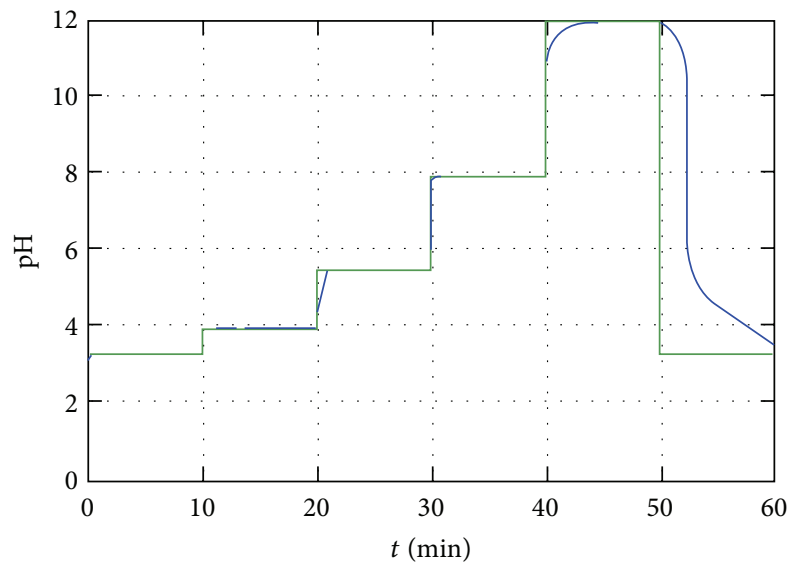

(c)

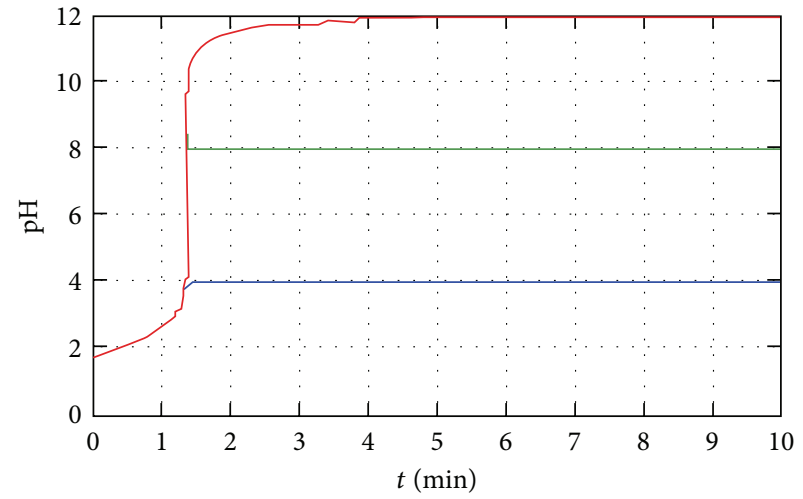

(b)

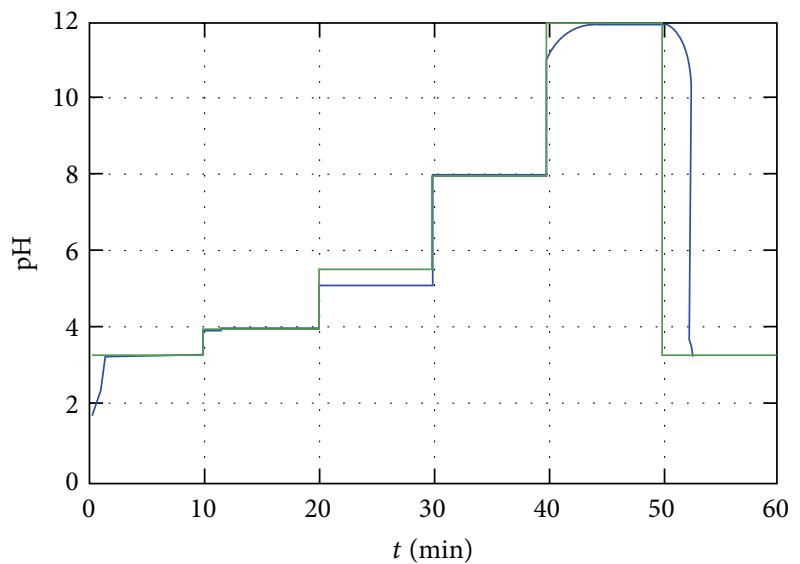

(d)

FIGURE 14: Performance of multiregional fuzzy PID controller in the three regions of titration curve for weak acid (a, c) and also for strong acid (b, d).

TABLE 2: Performance metrics for IAE.

\begin{tabular}{lccc}
\hline $\mathrm{pH}^{*}$ & Set point in percentage & PID & Multiregional fuzzy PID \\
\hline $7-8.4$ & $50-60 \%$ & 524.37 & 172.60 \\
$8.4-9.8$ & $60-70 \%$ & 333.54 & 124.32 \\
$9.8-11.2$ & $70-80 \%$ & 343.48 & 124.14 \\
$11.2-12.6$ & $80-90 \%$ & 342.08 & 163.82 \\
$12.6-7$ & $90-50 \%$ & 1881.85 & 857.95 \\
$7-5.6$ & $50-40 \%$ & 277.91 & 111.44 \\
$5.6-4.2$ & $40-30 \%$ & 293.88 & 118.08 \\
$4.2-2.8$ & $30-20 \%$ & 313.16 & 124.43 \\
$2.8-1.4$ & $20-10 \%$ & 355.36 & 137.14 \\
$1.4-7$ & $10-50 \%$ & 1755.76 & 749.34 \\
\hline
\end{tabular}

(ii) Two kinds of neural inverse models (MLP and RBF) are developed to simultaneously estimate the $\mathrm{pH}$ value. Two input parameters (base flow and temperature variation) are considered to train, test, and validate the proposed HNN structure. The obtained results ensure the higher accuracy and rapidity of the hybrid structure. The optimal structure of the proposed HNN estimator has been achieved for a set of readings containing 200 samples.
TABle 3: Performance metrics for ITAE.

\begin{tabular}{lccc}
\hline $\mathrm{pH}^{*}$ & Set point in percentage & PID & $\begin{array}{c}\text { Multiregional } \\
\text { fuzzy PID }\end{array}$ \\
\hline $7-8.4$ & $50-60 \%$ & 19627.06 & 10922.24 \\
$8.4-9.8$ & $60-70 \%$ & 19001.66 & 9622.48 \\
$9.8-11.2$ & $70-80 \%$ & 20021.90 & 10162.72 \\
$11.2-12.6$ & $80-90 \%$ & 2459.45 & 18886.27 \\
$12.6-7$ & $90-50 \%$ & 95529.10 & 59454.59 \\
$7-5.6$ & $50-40 \%$ & 12770.29 & 12271.11 \\
$5.6-4.2$ & $40-30 \%$ & 14849.38 & 13640.24 \\
$4.2-2.8$ & $30-20 \%$ & 17674.65 & 12579.83 \\
$2.8-1.4$ & $20-10 \%$ & 25622.83 & 12073.15 \\
$1.4-7$ & $10-50 \%$ & 100773.71 & 43639.44 \\
\hline
\end{tabular}

\subsection{Suggestions for Future Work}

(i) A rational controller could be added to manage the percentage in variation of base stream with respect to acid stream (e.g., weak or strong). Thus, the dynamics shown in Figure 14 can be further modified. 
(ii) Other nonlinear control strategies could be included into the module and chosen by the end-user to verify the performance of the plant and how it responds to different algorithms.

(iii) The proposed numerical and hybrid NN-based estimator could be realized using digital signal processor (DSP).

\section{Conflict of Interests}

The authors declare that there is no conflict of interests regarding the publication of this paper.

\section{Acknowledgment}

This project was supported by the Deanship of Scientific Research at Prince Sattam bin Abdulaziz University (Kingdom of Saudi Arabia) under Research Project no. 2014/01/2125.

\section{References}

[1] R. Ibrahim, Practical modeling and control implementation studies on a $\mathrm{pH}$ neutralization process pilot plant [Ph.D. thesis], Department of Electronics and Electrical Engineering, Faculty of Engineering, University of Glasgow, Glasgow, Scotland, 2008.

[2] S. Vaishnav and Z. Khan, "Design and performance of PID and fuzzy logic controller with smaller rule set for higher order system," in Proceedings of the International Conference on Modeling, Simulation and Control, pp. 855-858, San Francisco, Calif, USA, 2007.

[3] E. I. P. Volcke, Modeling, analysis and control of partial nitritation in a SHARON reactor [Ph.D. thesis], Ghent University, Ghent, Belgium, 2006.

[4] M. F. Zulfatman and M. F. Rahmat, "Application of self-tuning fuzzy PID controller on industrial hydraulic actuator using system identification approach," International Journal on Smart Sensing and Intelligent Systems, vol. 2, no. 2, pp. 246-261, 2009.

[5] N. Ebrahimi and A. Gharaveisi, "Optimal fuzzy supervisor controller for an active suspension system," International Journal of Soft Computing and Engineering, vol. 2, no. 4, pp. 36-39, 2012.

[6] S. Guan, H.-X. Li, and S. K. Tso, "Multivariable fuzzy supervisory control for the laminar cooling process of hot rolled slab," IEEE Transactions on Control Systems Technology, vol. 9, no. 2, pp. 348-356, 2001.

[7] I. Al-Adwan, M. Al Khawaldah, S. Asad, and A. Al Rawashdeh, "Design of an adaptive fuzzy-based control system using genetic algorithm over a $\mathrm{pH}$ titration process," International Journal of Research and Reviews in Applied Sciences, vol. 17, no. 2, pp. 177184, 2013.

[8] S. Asad, "Observing of $\mathrm{pH}$ for titration process with hybrid neural network structure," Journal of Engineering and Applied Sciences, vol. 6, no. 5, pp. 326-331, 2011.

[9] W. A. Agyare, S. J. Park, and P. L. G. Vlek, "Artificial neural network estimation of saturated hydraulic conductivity," Vadose Zone Journal, vol. 6, no. 2, pp. 423-431, 2007.

[10] D. J. Murray-Smith, "Simulation model quality issues in engineering: a review," in Proceedings of the 5th Symposium on Mathematical Modeling (MATHMOD '06), Vienna, Austria, February 2006.
[11] L. S. Saoud, F. Rahmoune, V. Tourtchine, and K. Baddari, "Modeling $\mathrm{pH}$ neutralization process using fuzzy dynamic neural units approaches," International Journal of Computer Applications, vol. 28, no. 4, pp. 22-29, 2011.

[12] F. J. Kohlmann, What Is pH, and How Is It Measured, A Technical Handbook for Industry, Hach Company, Loveland, Colo, USA, 2003.

[13] A. Gautam, S. Chatterji, and A. Kumar, "A review on temperature compensated $\mathrm{pH}$ monitoring and control system for process industries," International Journal of Scientific \& Engineering Research, vol. 4, no. 5, pp. 1707-1710, 2013. 

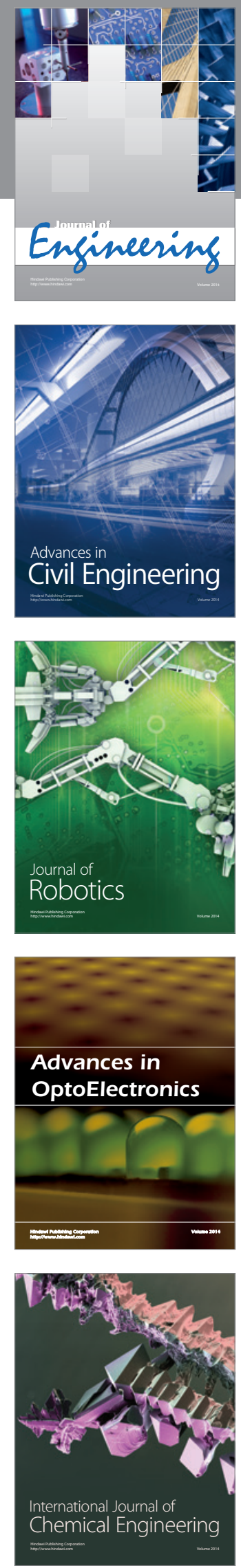

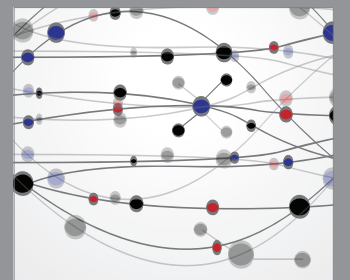

The Scientific World Journal
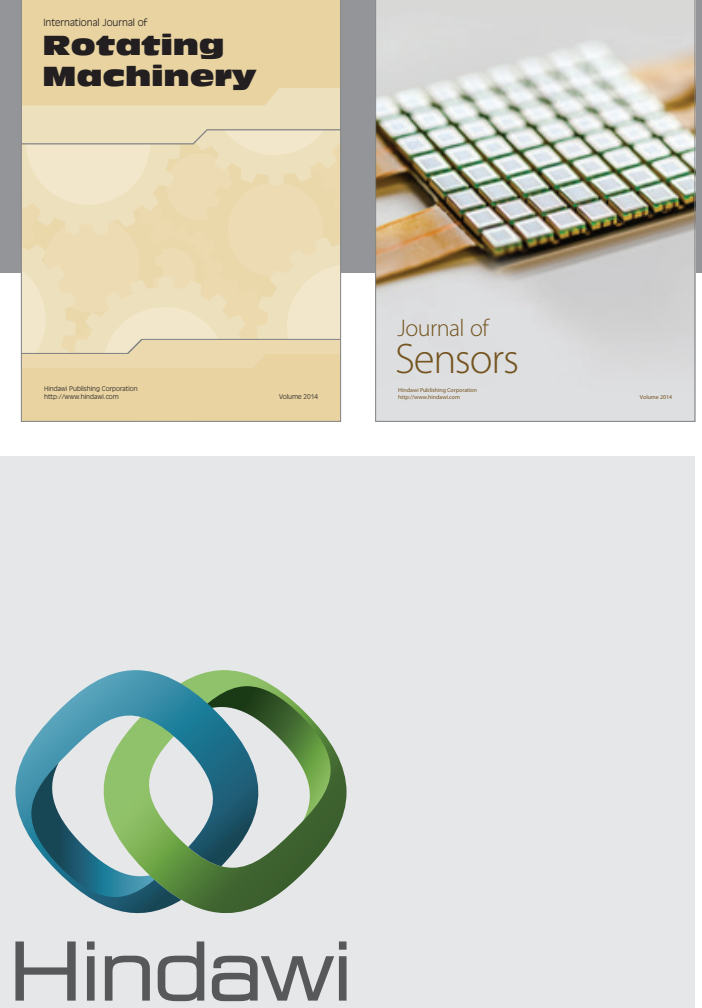

Submit your manuscripts at http://www.hindawi.com
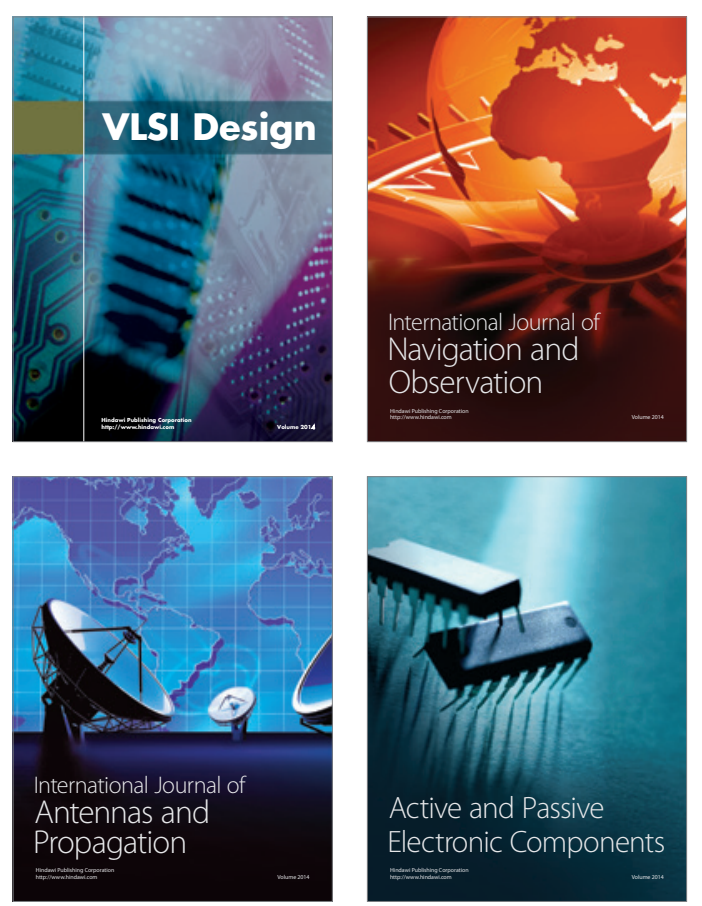
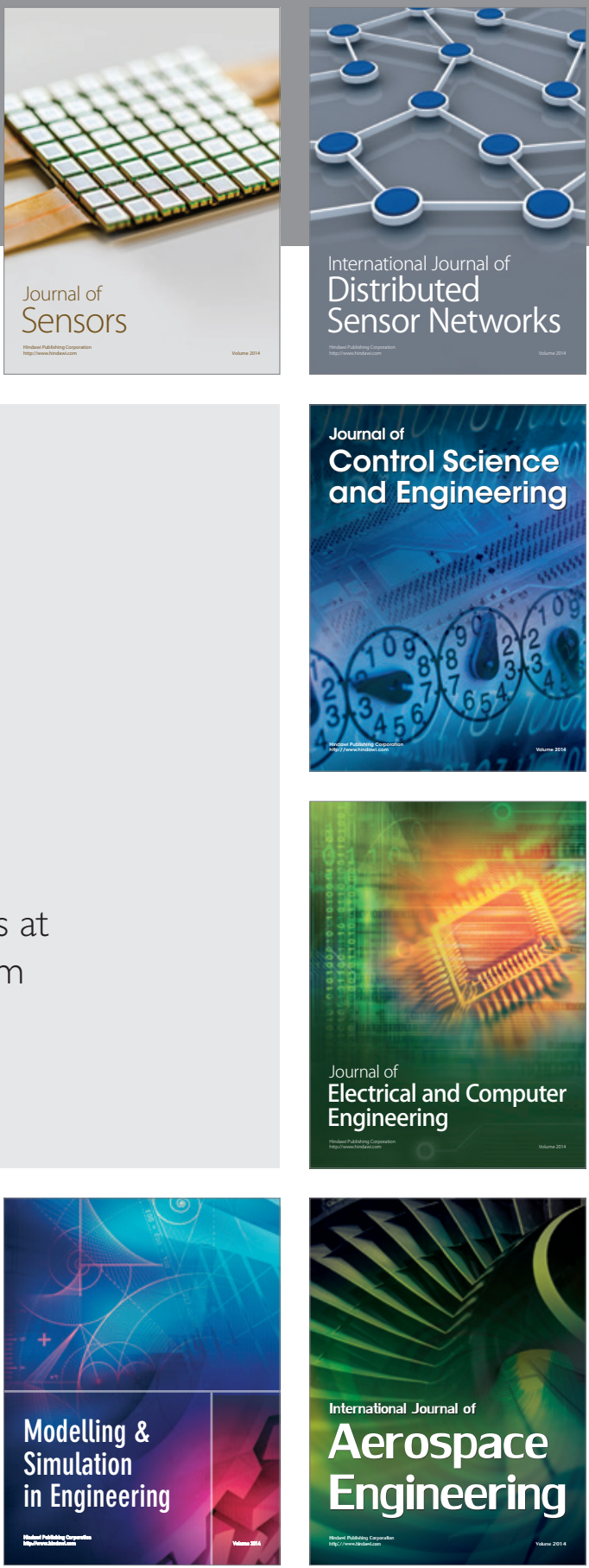

Journal of

Control Science

and Engineering
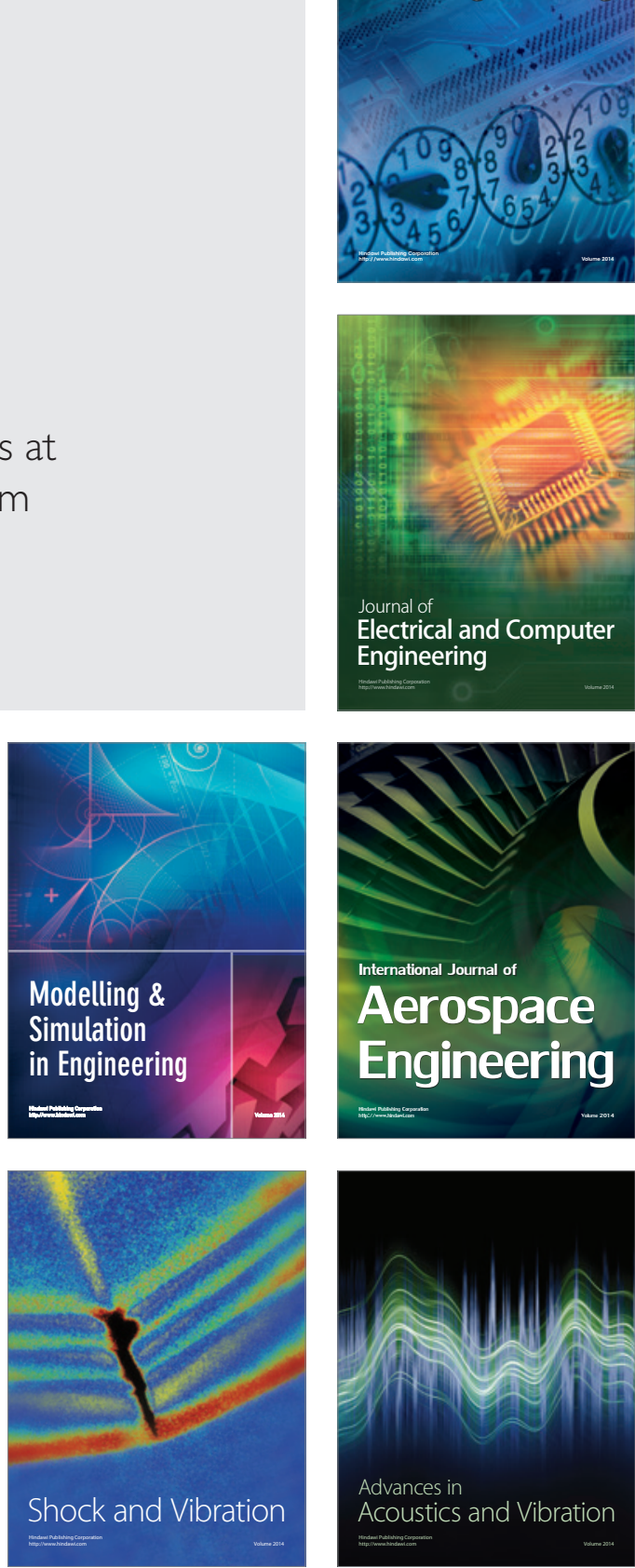